\title{
Comparison of levels and fusion approaches for multimodal biometrics
}

\author{
S. Sujana ${ }^{1}$, V. S. K. Reddy ${ }^{2}$ \\ ${ }^{1}$ Department of ECE, Jawaharlal Nehru Technological University, Hyderabad, India \\ ${ }^{1}$ Department of ECE, Vardhaman College of Engineering, Hyderabad, Telangana, India \\ ${ }^{2}$ Department of ECE, Malla Reddy University, Hyderabad, India
}

\begin{tabular}{l} 
Article Info \\
\hline Article history: \\
Received Jan 18, 2021 \\
Revised May 26, 2021 \\
Accepted Jun 1, 2021 \\
\hline Keywords: \\
Biometric recognition \\
Biometric authentication \\
Unimodal \\
Fusion levels \\
Multimodal biometric
\end{tabular}

Article Info

Article history:

Received Jan 18, 2021

Revised May 26, 2021

Keywords:

Biometric recognition

Unimodal

Multimodal biometric

\begin{abstract}
The biometric-based authentication system occupies maximal space in the field of security administration. Biometric applications are swiftly accelerating in day-to-day life such as computer login, smart homes, online banking, hospitals, border areas, industries, forensics, e-voting attendance system and investigation of crime. A reliable and accurate recognition body can be achieved with multimodal biometric methodologies. In this paper, we discuss starting with an introduction to biometric systems followed by their classification, and advantages as well as disadvantages. In today's world, most of the systems are unimodal biometrics having a lot of limitations to overcome those multimodal biometrics comes in to picture. In this paper we have discussed comprehensive representation on the system of multimodal biometric, various modes of undertakings, the significance of information fusion, a different section is allotted on the various possible levels of fusion involving sensor-level, feature-level, score-level, and decision-level as well as different rules of fusion.
\end{abstract}

This is an open access article under the CC BY-SA license.

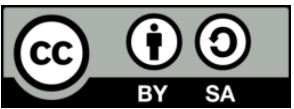

\section{Corresponding Author:}

S. Sujana

Department of Electronics and Communication Engineering

Research Scholar, Jawaharlal Nehru Technological University, Hyderabad, India

Email: sujanasurineni@gmail.com

\section{INTRODUCTION}

Biometrics is a way to measure a person's physical characteristics to authenticate their identity. The dominant theme of the biometric authentication system is to identify an individual based on their unique traits (physical or behavioral) [1]. The individual behavioral characteristic contains how the person unique qualities like allies and action, such as their uttered manner, body pantomime, signature and voice. The physiological class yields physical being's attributes such as palm print, face, iris, fingerprints, and many more. To figure out these attributes assists the process of recognition using the biometric administration [2]. Conventional identification approaches differentiate people based on susceptible passwords or magnetic/ID cards. These key identifiers are prone to misutilization by unauthorized persons once they have them in hand [3]. Common issues with the conventional approaches are stealing, forgetting, losing, which make it capricious and unsound in the immensely accurate system like banks, forensics, and ports systems [4].

In this generation, there is a diversified experience that people need to validate themselves. Validation is a process to govern that somebody is certainly the person that he proclaimed to be or not. Predominantly, there are three types of validation they are something you know (password) second one something you have (token), and the final one is something you are [5], [6]. People can validate themselves without remembering the intricate mix or carry any implement. People need only their attributes to validate 
themselves, for example, their fingerprint, eyes, retina and hand [7]. This type of authentication method is called biometrics.

The foremost advantage of biometrics when compared to other methods, it cannot be vanished or stolen. Due to the sky-scraping performance of biometrics, it became an essential and preferred one to understand and interpret human attributes for security. This makes spoofing difficult [8]. This paper elaborates on two types of biometric systems namely multimodal and unimodal, focus the issues related to unimodal systems. Following sections of this paper: section 2, describes review criteria; section 3 is the process involved in a general biometric system. Section 4 talks about the classification of biometric systems. Section 5 discussed the levels of fusion in multimodal systems, while section 6 , highlights the methods of fusion in multimodal biometrics, section 7 discussion, and section 9 concludes this paper.

\section{REVIEW CRITERIA}

Chen et al. [9] suggested a comprehensive face template protection scheme to secure the original face template. The facial feature of each is mapped to a different binary code in the training using deep multilabel learning. In the recognition process, CNN output is decoded with an LDPC decoder to suppress Gaussian noise caused by intra-variations. The results showed that higher GARs could be achieved by the proposed scheme. To enhance the accuracy of the proposed system, it cannot map the facial features to highdimensional LDPC codes.

Hamd and Ahmed [10] implemented an iris recognition system by using two approaches: i.e., principal component analysis and Fourier descriptors. In Fourier descriptors, iris features are extracted in the frequency domain (FD). Statistic technique used by the principal component analysis to select the important feature values for reducing dimensionality and finally three various distance measurement methods used for comparison. In matching results always Fourier descriptors were advanced with $96 \%, 94 \%$, and $86 \%$ correct matching against $94 \%, 92 \%$, and $80 \%$ for principal component analysis using Manhattan, Euclidean, and Cosine classifiers, respectively. They concluded that Manhattan achieves the best results from Euclidean and Cosine in FD and PCA.

Ammour et al. [11] proposed a multimodal scheme for biometric authentication based on the iris and face. They used fusion at score level with different fusion rules normalization techniques. Face ORL database and CASIA-V3-Interval database used to validate the proposed system. The results showed that their proposed optimal system having a good recognition rate of $98 \%$.

Ammour et al. [12] a multi-modal face-iris framework based on texture information using 2D Log Gabor filter in combination with spectral regression kernel discriminant analysis (SRKDA) is proposed to extract features and minimize the dimensionality of the extracted features from modalities. They selected hybrid-level fusion to extract the advantages of different fusions and using database CASIA Iris Distance achieved up to $0.24 \%$ improvement of EER when compared to the unimodal

Sujana and Reddy [13] developed the optimal multimodal device for iris and face by properly choosing features and scores these optimized trait data affect the efficiency of the device. At different fusion stages, they analyzed several techniques to find an effective technique for merging face and iris then integrating the advantages of multiple fusion techniques to create a stable combined device. CASIA Iris Distance Database verification results with GAR $=93.91$ percent with FAR $=0.01$ percent. Showing major advances over unimodal and multimodal fusion methods in the suggested mixed fusion scheme

Matin et al. [14] Selected a fusion method for the weighted score level in a multimodal biometric system to combine iris and face scores. They employed Daugman's technique for Iris recognition and the PCA technique for face recognition. They used the Min-max normalization technique to balance the facial and iris scores. Finally, to combine their normalized scores weighted sum rule was used. This will provide better results than a unimodal system.

Azom et al. [15] have presented a hybrid fusion process by combining three levels of fusion such as feature, score, and decision using decision rule. To get the fused classifiers, they performed a feature-level fusion for the face and Iris. Then the weighted fusion of the score level between face LDA and Iris using $\mathrm{LBPH}$, but individual modalities generated the highest recognition rate. They obtained a $98.75 \%$ recognition rate when validated using the CASIA iris and ORL face.

Huo et al. [16] established a Multi-modal system of feature level face-iris .2D Gabor filter bank used to extract the features of both the modalities, these features are converted using histogram statistics. The fusion recognition depends on support vector machine and principal components analysis. Their results show that it effectively extracts iris and face features as well as offers higher accuracy of identification.

Eskandari and Toygar Ö [17] proposed a framework for iris-face modalities based on score and feature-level fusion. To get the iris features iris 1D Log- Gabor filter was used and a backtracking search algorithm (BSA) is used to obtain the optimized features used in feature level fusion and optimized weights 
assigned to scores in score level fusion to achieve an efficient authentication system at the feature and matching score levels. In contrast with unimodal and other multimodal methods, a major increase in identification was achieved.

Khiari-Hili et al. [18] suggested a multi-modal system using iris and face. They explored two approaches for combining the scores at score-level fusion. Initially, A single joint quality metric of a galleryprobe comparison based on iris occlusion. Then, they placed in the weighted sum fusion to dynamicallycontrol the weights. The fusion rule increases protection by reducing error rates in uncontrolled environments compared to sum and weighted sum laws by two suggested quality metric strategies. They suggested a framework on the MBGC database in the future with more quality measures related to face.

Minaee et al. [19] developed a face recognition system based on scattering convolutional architecture, scattering transform technique for feature extraction, and SVM for classification. However, Scale-invariant scattered features can be used for improvement inaccuracy, which they didn't use here.

Sharifi and Eskandari [20] have enlightened us with the facts of three fusions levels (feature, score, and decision) to efficiently combine input traits such as face and iris. He has used the log-Gabor transformation for extraction of iris and face features which are combined to construct a robust and optimized scheme particularly fusion at the decision level in the proposed one. To improve recognition accuracy, he applied the backtracking Search algorithm by selecting the optimized weights at the score level and reducing features at feature level fusion.

Ahmadi and Gholamreza [21] have proposed a method to increase the performance by human recognition system multi-layer perceptron-based and particle swarm optimization, whose combination is considered as a classifier where the features are extracted using the 2-D Gabor filter and their obtained accuracy was 95.36 which is not very high as compared to many models nowadays.

Ammour et al. [22] proposed a system using hybrid level fusion in which is a face and iris as input modalities. The 2D log Gabor filter is used for the extraction of the face and left and right iris characteristics. The database of the CASIA iris distance is used to test the proposed method and concluded that it achieves an improvement up to $0.24 \%$ to EER than the previous.

Dua et al. [23] suggested a feed-forward architecture and uses a k-means clustering algorithm to distinguish iris patterns. For iris and pupil boundary localization, an integro-differential operator along with a circular Hough Transform is used. Daugman rubber sheet model for Iris normalization and the 1D Gabor filter to extracting feature. But the system could not perform well under various environments. Table 1 presents the literature related to the different existing techniques for feature extraction, classification, and fusion methods at various levels of fusion in multi-model biometrics.

Table 1. A glance of existing techniques in multi-model biometrics

\begin{tabular}{|c|c|c|c|c|}
\hline Author & Feature Extraction & Level of fusion & Fusion method/Classifier & $\begin{array}{l}\text { Accuracy } \\
(\%)\end{array}$ \\
\hline Rohit Agarwal [24] & $\begin{array}{l}\text { Gray -Level Co-occurrence Matrix } \\
\text { \&NeighborhoodGray-one Difference Matrix }\end{array}$ & $\begin{array}{l}\text { Decision level/ } \\
\text { D-S theory }\end{array}$ & SVM & 97.8 \\
\hline $\begin{array}{l}\text { Ahmed Shamil } \\
\text { Mustafa [25] }\end{array}$ & $\begin{array}{l}\text { Gray -Level Co - } \\
\text { Occurrence Matrix (GLCM) with KNN. }\end{array}$ & Decision fusion & AND gate & 95 \\
\hline $\begin{array}{l}\text { Lemmouchi } \\
\text { Mansoura [26]. }\end{array}$ & FFT (face, iris), SVD (face, iris) & Score level & $\begin{array}{l}\text { Min rule with } \\
\text { Prctilenormalization } \\
\text { /Euclidean distance }\end{array}$ & $\begin{array}{l}98.33 \\
94.17\end{array}$ \\
\hline J. Raja [27] & Gabor wavelet transformation & $\begin{array}{l}\text { ESVM-KM } \\
\text { technique }\end{array}$ & Ensembled SVM Classifier & 93.15 \\
\hline BasmaAmmour [28] & $\begin{array}{l}\text { Multi-resolution 2D Log-Gabor filter singular } \\
\text { spectrum analysis, Normal inverse Gaussian } \\
\text { combined with statistical features of wavelet. }\end{array}$ & $\begin{array}{l}\text { Hybrid fusion } \\
\text { level (score and } \\
\text { decision) }\end{array}$ & $\begin{array}{l}\text { Max rule with Min-Max } \\
\text { normalization }\end{array}$ & $\begin{array}{l}99.16 \\
99.33\end{array}$ \\
\hline VedururuSireesh [29] & Modified LBP & $\begin{array}{l}\text { Feature fusion } \\
\text { Score fusion }\end{array}$ & $\begin{array}{l}\text { PSO and naive bayes } \\
\text { classifier }\end{array}$ & $\begin{array}{l}90 \\
85\end{array}$ \\
\hline $\begin{array}{l}\text { Sheetal Chaudhary } \\
\& \text { RajenderNath [30] }\end{array}$ & $\begin{array}{l}\text { Face- Eigenface approach. } \\
\text { Fingerprint -minutiae points }\end{array}$ & $\begin{array}{l}\text { Match score } \\
\text { level fusion }\end{array}$ & $\begin{array}{l}\text { Multiple support vector } \\
\text { machines (SVMs) }\end{array}$ & $\begin{array}{c}99.02 \\
99.8\end{array}$ \\
\hline $\begin{array}{l}\text { Suneet Narula Garg } \\
\text { [31] }\end{array}$ & $\begin{array}{l}\text { Coarseness, Contrast, Directionality, Entropy, } \\
\text { Homogeneity and Energy }\end{array}$ & $\begin{array}{l}\text { Decision level } \\
\text { fusion }\end{array}$ & KNN and Neural classifier & 91.5 \\
\hline Archana P. Patil [32] & $\begin{array}{l}\text { Minutiae extractor2D Gabor filter } \\
\text { haar wavelet transform }\end{array}$ & $\begin{array}{l}\text { Match score } \\
\text { level fusion }\end{array}$ & $\begin{array}{l}\text { weighted fusion } \\
\text { technique/KNN }\end{array}$ & 95.23 \\
\hline
\end{tabular}

From the above Literature survey, we can conclude that most of the authors have worked on the conventional feature extraction and classification techniques which led them to a loss of accuracy. To improve the accuracy, the use of advanced methodologies is required, for example, convolutional neural network $(\mathrm{CNN})$, pre-trained networks for feature extraction, and neural network for classification. 


\section{PROCESS INVOLVED IN BIOMETRIC SYSTEMS}

Generally, a biometric system mainly consists of two phases namely the enrollment stage and confirmation stage. In the enrollment phase, the images are collected from biometric attributes and it is processed to get a clear image as well as to rectify distortions and to get the sector of interest for feature extraction. Certain features alone are extracted from the image to form the feature vector and are then stored in a database [33] shown in Figure 1 to recognize particular individuals.

In the acceptance phase, the query image which is to be tested is processed to improve the hallmark of an image. The Region of Interest is the procedure of emphasis key and required features in a biometric feature as an interesting region that will further be used as matching parameters and then feature extracted. Thus, formed feature vectors from retrieved features will be compared against the feature database in the matching module to produce a match score and finally by using the match score decision module will identify the authorized persons. These sequences of steps are shown in Figure 1. A biometric system can be represented with two important functionalities one is verification and the other one is identification [34]. Verification involves a one-to-one match in the database. On the other hand, identification is obtained when a system performs one to many comparisons.

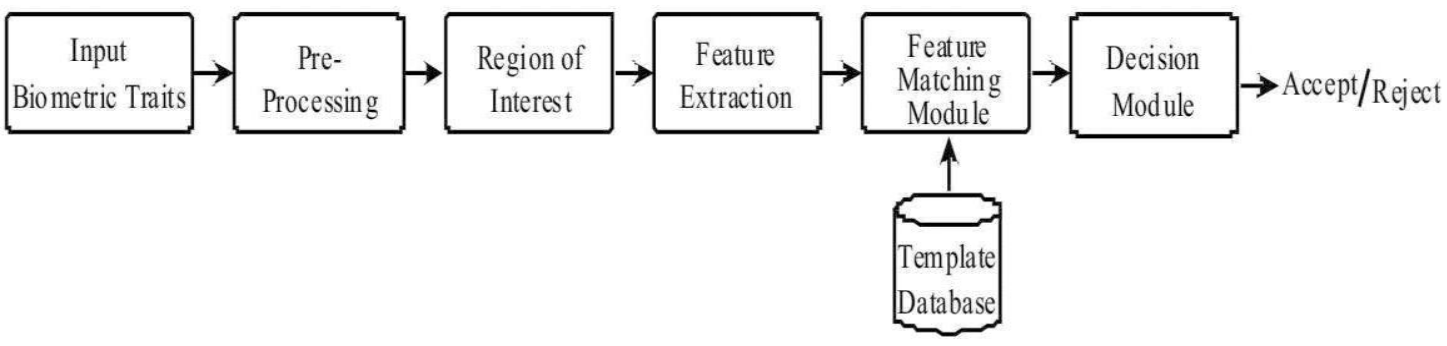

Figure 1. Steps associated with a biometric system during recognition

\section{CLASSIFICATION OF BIOMETRIC SYSTEMS}

\subsection{Unimodal biometric systems}

Biometric authentication of individuals is by using their behavioral or physiological features. These biological features are organized into unimodal and multimodal biometric systems [35]. Many of the biometrics are unimodal systems, which means it employs single biometric attributes to recognize the user are normally cost-efficient, but the performance of these systems may degrade in some practical circumstances were there existing noisy data, intra-class variations, and inter-class similarities.

Though some unimodal systems have made a substantial improvement in accuracy and reliability, they usually experience problems in the enrollment stage due to the non-universality of biometric attributes. In Biometric spoofing unauthorized person can try to imitate behavioral biometrics like voice and signature for an enrolled user. However, inadequate accuracy caused by noisy data that occurred while captured biometric data usually contains incomplete acquisition conditions or variants in a characteristic of biometric itself like un-wanted scratches on the biometric image or dirt on sensor [36].

Intra-class variation: The biometric data collected during authentication will not be the same as the one used for generating a template for an individual during the enrollment process. Inter-class similarities: It because of overlapping feature spaces in the feature sets of various users. The unimodal biometric system may lead to both false rejection rate (FRR) and false acceptance rate (FAR) [18].

\subsection{Multimodal biometric systems}

Unimodal biometric systems constantly fail to correctly authenticate an individual with a craving effect and accuracy. However, multimodality (more than one trait) is applied to resolve many of the issues related to unimodal systems. The term 'multimodal' is utilized to describe the mix of at least two different biometrics of a person (i.e., iris, face, and fingerprint) sensed by using distinct sensors and thus improve the required accuracy of a biometric system by utilizing the necessary information from multiple input modalities.

It may be a fusion of behavioral with physical modalities or different physiological traits together. These fusion methodologies decrease the effect of spoofing attacks by making it difficult for an unauthorized one to fake, copy or steal, raises the degree of freedom, decreases the failure-to-enroll rate, and hence makes the biometric system more secure. Increasing the discriminate information leads to reduce the error in the recognition process [37]. Multimodal biometric's fusion techniques mention how the information is merged 
when it's obtained from various biometric traits. This fusion can be done at single as well as multiple levels in a multimodal system, i.e., fusion at the sensor, at feature level and matching-score, or decision level [21]. Fusion at the feature level, however, is more fruitful because it contains more information about the input trait than the levels of fusion after matching [11].

To acquire the target of claimed performance improvement, the fusion rule chosen should depend on the type of applications that are selected, input biometric modalities used for fusion, and the opted fusion level. Multimodal systems can mix information at various stages, but the fusion at score level became the most favored one. Fusions also address the problem of spoofing and non-universality. Several studies have suggested that integrating information from various biometrics and improved accuracy to satisfy the specifications of the physical world [38]. Hence, the multimodal biometric system shows several benefits than a unimodal biometric.

\section{LEVELS OF FUSION IN MULTIMODAL BIOMETRIC SYSTEM}

Multimodal fusion can be accomplished in two ways.

\subsection{Fusion just before matching} fusion level.

It is possible to acquire fusion precedent to matching in two distinct manners: sensor level and

\subsubsection{Sensor level fusion}

Sensor-level fusion integrates the data that is obtained from multiple sensors and gives fused information [10] and from these fused data features can be pulled shown in Figure 2 (a). Different methods in the sensor level are single sensor multiple instances:

- Several instances achieved from a single sensor are combined here to obtain the complete data.

- Intraclass multiple sensors: Several instances discovered from different sensors are put together to identify the details in this situation. Inter-class multiple sensors: The data to be fused must be oblique to be of the same kind, like two images that will be fused from two separate cameras required for the same resolution.

- Sensor-level fusion addresses the noise in sensed data due to not proper maintenance of sensors. This fusion has not received much attention since it has more redundant information [39].

\subsubsection{Feature level fusion}

The fusion of the feature level is acquired by joining various feature sets obtained from multiple biometric sources [12], [40] shown in Figure 2 (b). Sets of features can be either homogeneous or heterogeneous. When distinct methods are used for one feature extraction, non-uniform feature vectors are achieved, or feature vectors are extracted from various modalities. The process of fusion cannot be complete successfully if the feature vectors utilized are not coherent with one another, like mixing of fingerprint minutiae with Eigenface coefficient is not possible.

The mechanism involved in feature level fusion evolves in two steps, i.e., the normalization of an extracted feature and then the selection of a feature. The feature sets are first translated into a generic domain, and the range of feature sets are altered, this can be implemented by using normalization techniques [41].

Linear discriminate analysis (LDA) was applied in the feature drawing phase to solve the issue of a large proportion of the combined features. Techniques such as PCA or sequential backward selection, forward sequential selection, are utilized to minimize the dimensions of a feature set. The amount of information at the feature level is sufficient to authenticate an individual; however, the feature-level fusion is hard because feature sets of various sources may either be incompatible or inaccessible [42].

\subsection{Fusion just after matching}

It is a fusion after comparison of the extracted features with stored template database can be achieved in two ways: fusion at score level and decision level.

\subsubsection{Match score level fusion}

To generate match scores, feature vectors are extracted separately for an individual biometric trait and these feature vectors are compared with templates stored in the database during enrollment [12]. The score given by the matchers has the required information regarding input and also its feature vector representation. Set of outputs from matching module i.e., match scores are merged to create a single scalar score shown in Figure 3 (a).

The acquired scores from matching modules cannot be integrated directly since the scores obtained from different modalities have different ranges. It is important to convert these scores to a common domain or 
scale by using Score normalization to ensure proper mixing of scores from the various modalities [10], [13]. Fusion at the score level is usually favored because the scores supplied by the individual matching modules are easily accessed and incorporated. The information accessible at the score level is adequate to recognize an individual client since it has neither an excessive amount of repetitive nor too little data. It gives a strong set of information [43]. It is simple to aggregate the scores produced by several matchers here. This method of fusion is the most commonly used.

\subsubsection{Decision level fusion}

In this fusion, the combining of multiple score information is captured from various biometric modalities when the individual decision module gives its decision regarding the identity of a person of claimed. In this decision fusion the final classification result depends on the outputs of the decision modules corresponding to various modalities see in Figure 3 (b) and the final score is classified into one of the two (reject or accept) main classes [44]. This Fusion is too rigid because it has less information to make a decision. Commercial off the Shelf tools give the final decisions by using some of the techniques like majority voting, Bayesian decision fusion, AND and OR. Generally, the most used approach for authentication is majority voting for decision level fusion. The benefit of this method is that here prior knowledge of the matcher does not require as well as any need of training to take a final decision. Sometimes performance degradation may occur in 'AND' and 'OR' methods because of the mixing of multiple matchers. The fusion at the decision is only used where the decisions of the individual users are accessible hence is known as an abstract level fusion [45]. Comparative analysis of of various levels of fusion [46] is shown in Table 2.

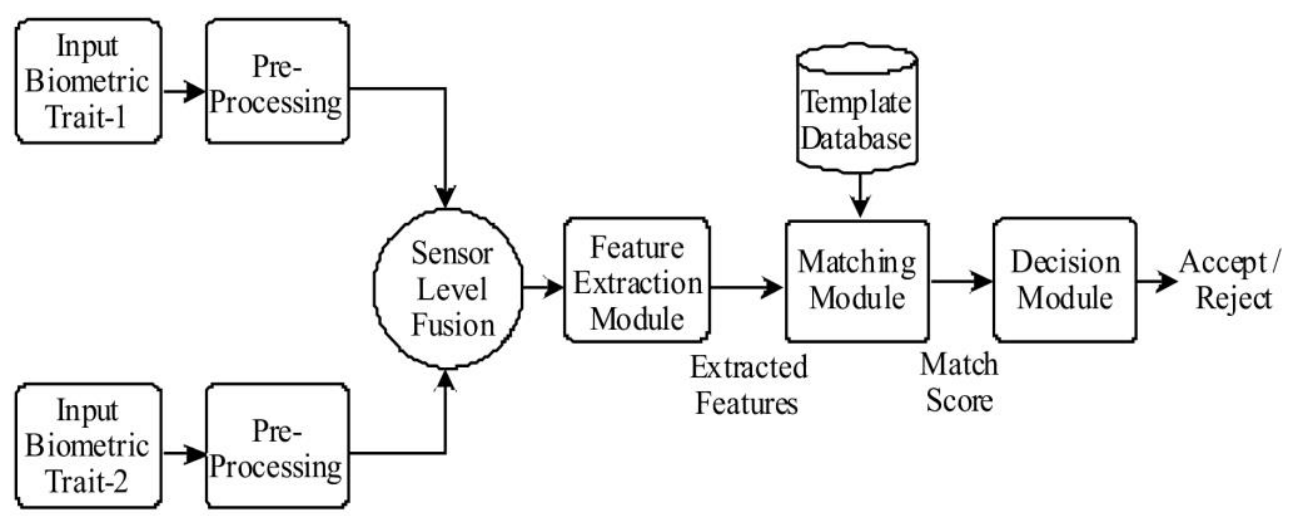

(a)

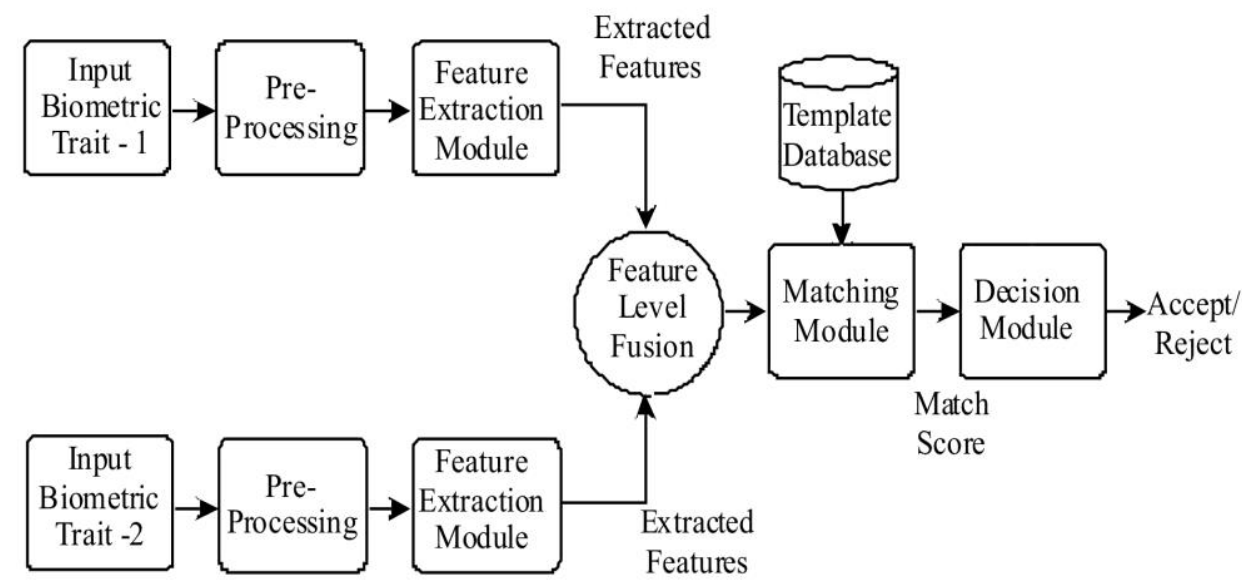

(b)

Figure 2. Fusion process at; (a) sensor level of two biometric traits and (b) feature level of two biometric traits 


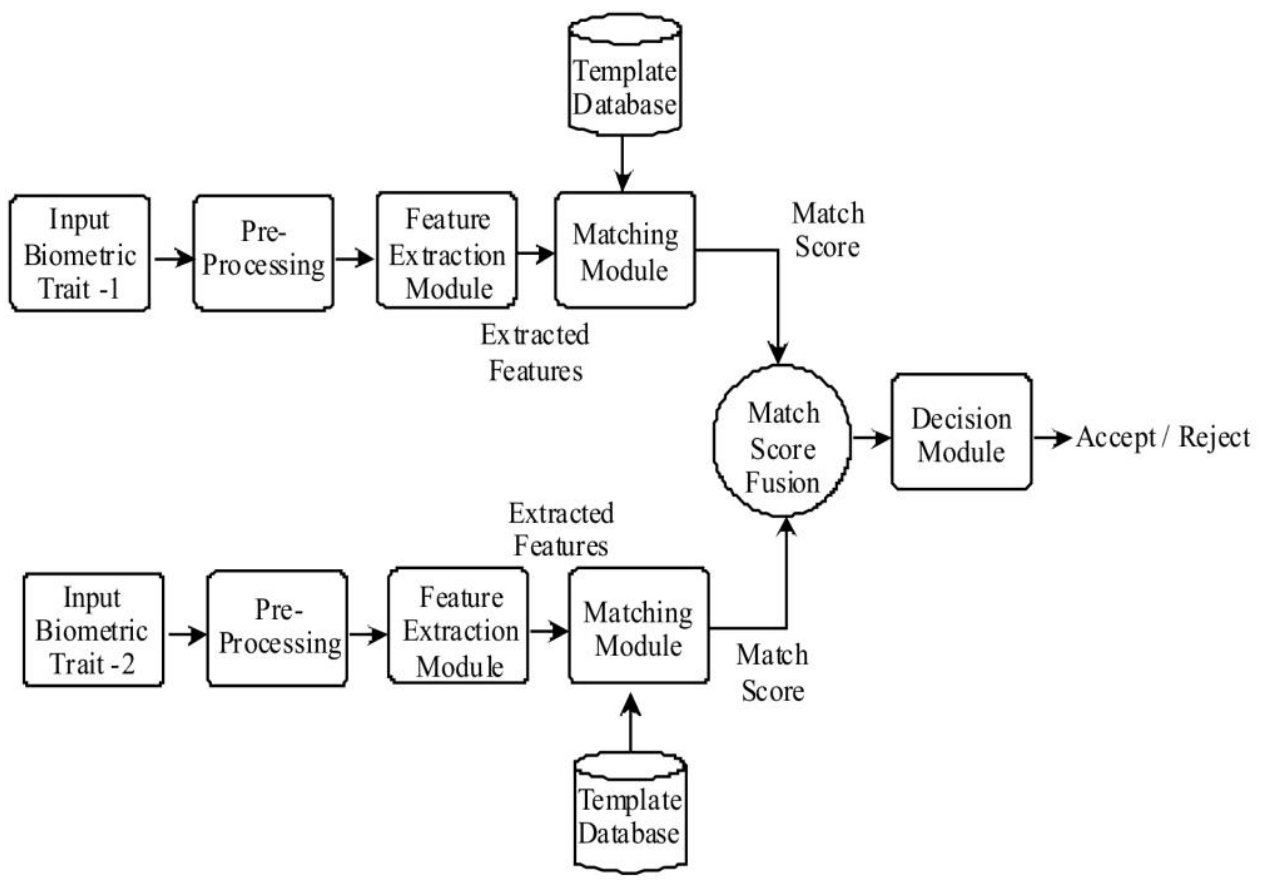

(a)

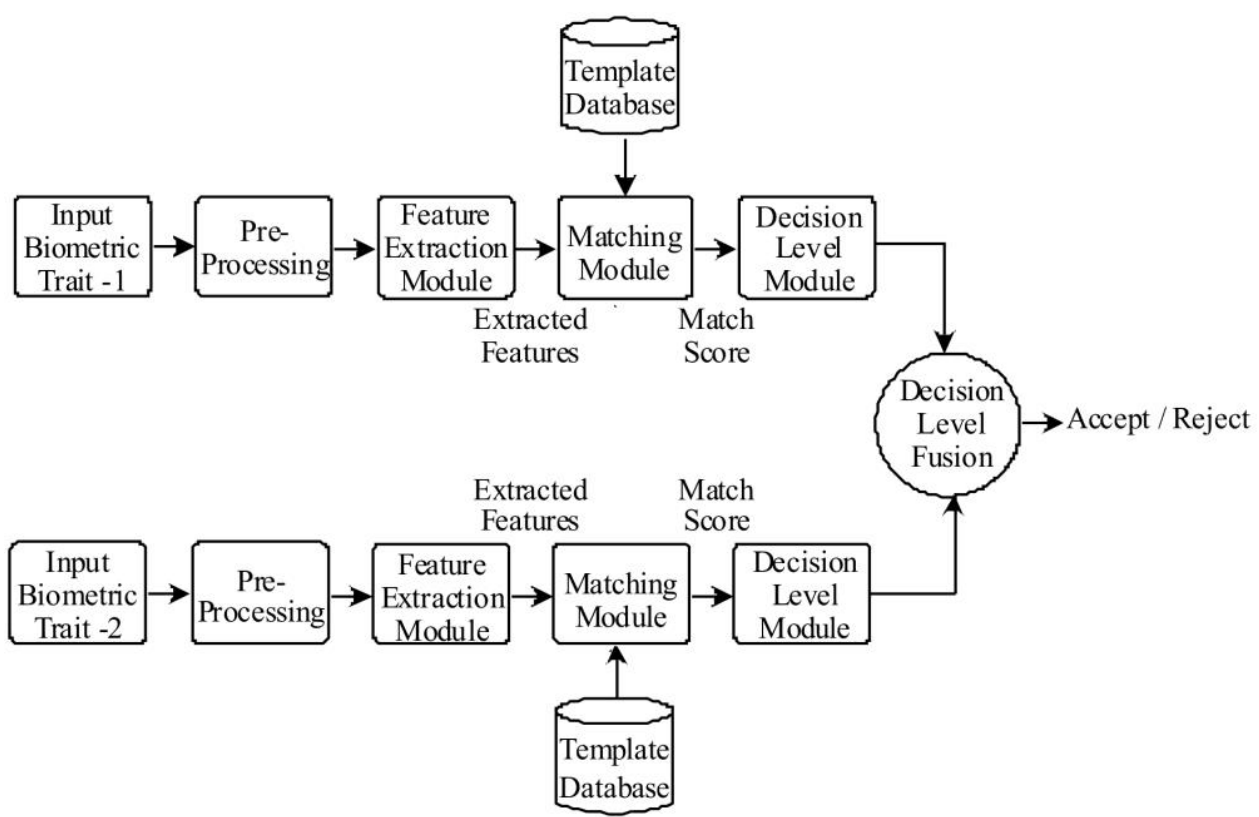

(b)

Figure 3. Fusion of; (a) two biometric traits at the score level and (b) biometric traits at the decision level

Table 2. Comparative analysis of various levels of fusion

\begin{tabular}{ll}
\hline \multicolumn{1}{c}{ Fusion level } & \multicolumn{1}{c}{ Limitations } \\
\hline $\begin{array}{l}\text { Fusion at the } \\
\text { Sensor level }\end{array}$ & The noise of sensed data, low sensor efficiency, and atmospheric influences. \\
$\begin{array}{l}\text { Fusion at feature } \\
\text { Incompatible feature collection, the uncertain relationship between various biometric systems' feature spaces, } \\
\text { which require considerably more complex matching. }\end{array}$ \\
$\begin{array}{l}\text { Fusion at match } \\
\begin{array}{l}\text { There are no homogenous scores obtained from various matchers. It is not necessary that the scores obtained should } \\
\text { Fusion at score } \\
\text { level }\end{array}\end{array}$ & $\begin{array}{l}\text { be within the same scope. It is important to apply normalisation schemes } \\
\text { Only a minimal amount of knowledge at this stage of fusion is available. }\end{array}$ \\
\hline
\end{tabular}




\section{METHODS OF MULTIMODAL FUSION}

There are three main categories for the classification of fusion methods for multimodal biometrics, such as estimation based, classification based and rule-based methods [47].

\subsection{Rule-based fusion techniques}

The rule-based techniques in multimodal are involving some basic rules for fusing information. In this case, some statistical rules are used such as MIN, MAX, product, and the sum-based fusion like linear weighted, the majority voting, OR and AND [48]. These techniques are depending on the selected application and are customized. Generally, these methods perform well if the temporal alignment of traits is of good quality. In rule base there is no training process are also known as unsupervised methods, but the learning or training rules are mostly applicable for pre-defined output.

\subsection{Classification based fusion methods}

Fusion based on classification is a supervised category as it is based on training or learning process. This classification method involves a set of techniques to classify the observations into one of the predecided classes. The most commonly used classification methods are neural networks, maximum entropy models, Dempster-Shafer theory, dynamic Bayesian network, support vector machine, and Bayesian inference. From a machine learning point of view, it is further classified into two ways namely generative and discriminative methods. Bayesian inference and dynamic Bayesian network come under generative, whereas neural networks and supportive vector machines are under Discriminative models. Neural network and Bayesian method are applicable to feature level as well as decision level fusion methods [49].

\subsection{Estimation-based fusion techniques}

To estimate the location of objects moving based on multimodal data, estimation fusion is used. In a recognition process of a person, multiple input modalities are combined to estimate the location. The particle filter and Kalman filter belong to these fusion methods. Kalman filter allows low-level dynamic data to be performed in real-time and calculates the state of the system from fused data [50]. Particle-based approach or Sequential Monte Carol method is simulation-based methods involves prediction and update steps. Based on the information of multimodal these techniques are used for an estimation of the state of moving.

\section{DISCUSSIONS}

The review clarifies that still there is a need to investigate more about the problems existing in various biometric recognition systems and various fusion methods. Few challenges which are currently prevailing in various biometrics can be explained through few examples. Firstly, in fingerprint-based recognition systems, the key attributes are the features that tend to deteriorate as one becomes older. Secondly, the voice recognizing system might be problematic if the enrolled person loses their voice, then it may lead to difficulty in identification. Thirdly, if a person suffers from arthritis, then he /she might have difficulty being authorized on a hand geometry base system. Moreover, in a face recognizing system major problem faced is due to the disparity in features caused by various factors like facial expression changes, illumination changes, and majorly due to occlusion. Finally, one of the most reliable iris recognition systems also faces recognition issues due to the eyelashes, lenses, and reflections from obstacles. Furthermore, because of the number of challenges and issues, the accuracy of the sensing systems is becoming low which stimulates the interest to work in this area. To overcome the above-mentioned problem faced in unimodal biometric systems we suggest using multimodal biometrics. The key factor available in multimodal biometrics is the fusion techniques. As per our review, we have come across a wide range of fusion techniques, a proper combination of these existing fusion techniques leads to the solution for the abovementioned problems. The neural network along with convolutional neural network (CNN) with proper processing and fusion techniques solves the issues faced by the biometric systems.

\section{CONCLUSION}

A research-based review is performed on a Biometric-based authentication system. In this paper, a detailed study of biometrics starting from traditional security to the recent multimodal biometric systems has been done. We have discussed two main classifications of biometric systems i.e., unimodal, and multimodal systems. By the imperfection of unimodal and other problems, the multimodal recognition system has been introduced. The various methods and levels of fusion available in multimodal systems were also covered. This paper gives clarity that there's a huge scope of improvement to identify the solutions to the issues observed in the various biometric recognition systems also in the different levels of fusion as well as various 
methods of fusion. Multimodal biometrics is an exciting and interesting research area that makes a fusion of sources at various levels for better accuracy, security, and reliability. The applications and need for Multimodal biometric would be an integral part of the future generation of any technology.

\section{REFERENCES}

[1] Lahmidi A., Minaoui K., and Rziza M., “A robust minutia-based approach for securing fingerprint templates," 2018 9th International Symposium on Signal, Image,Video and Communications (ISIVC), 2018, pp. 286-290, doi: 10.1109/ISIVC.2018.8709184.

[2] Liakat Ali M., Monaco J. V., Tappert C. C., and Qiu M., "Keystroke biometric systems for user authentication," Journal of Signal Processing Systems., vol. 85, no. 2-3, pp. 175-190, Mar. 2017, doi: 10.1007/s11265-016-1114-9.

[3] Kumar T., Bhushan S., and Jangra S., "A Brief Review of Image Quality Enhancement Techniques Based Multimodal Biometric Fusion Systems," International Conference on Advanced Informatics for Computing Research, 2018, pp. 407-423, doi: 10.1007/978-981-13-3140-4_37.

[4] Pathak M. and Srinivasu N., "Performance of Multimodal Biometric System Based on Level and Method of Fusion," Chakrabarti A., Sharma N., and Balas V. E., Advances in Computing Applications, New York, USA: Springer, 2016, pp. 137-152, doi.org/10.1007/978-981-10-2630-0_9.

[5] Buciu I. and Gacsadi A., "Biometrics systems and technologies: A survey," International Journal of Computers, Communications and Control, vol.11, no. 3, pp. 315-330, 2016, doi: 10.15837/ijccc.2016.3.2556.

[6] Sayeed S, Nasir I, Ong T. S, "An Efficient Multimodal Biometric Authentication Integrating Fingerprint and Face Features," American journal of applied sciences, science publications., vol. 13, no. 11, pp. 1221-1227, 2016, doi: 10.3844/ajassp.2016.1221.1227.

[7] Elhoseny M., Elkhateb A., Sahlol A., and Hassanien A. E., "Multimodal biometric personal identification and verification," Hassanien A. E. and Oliva D. A., Advances in Soft Computing and Machine Learning in Image Processing, New York, USA: Springer, pp. 249-276, 2018, doi: 10.1007/978-3-319-63754-9_12.

[8] Yang W., Wang S., Hu J., Zheng G., and Valli C., "Security and accuracy of fingerprint-based biometrics: A review," Symmetry, vol. 11, no. 2, 2019, doi: 10.3390/sym11020141.

[9] Chen L., Zhao G., Zhou J., Ho A. T., and Cheng L. M., "Face template protection using deep LDPC codes learning," IET Biometrics, vol. 8, no. 3, pp. 190-197, 2018, doi:10.1049/iet-bmt.2018.5156.

[10] Hamd M. H and Ahmed S. K, "A Biometric System for Iris Recognition Based on Fourier Descriptors and Principle Component Analysis," Iraqi Journal for Electrical And Electronic Engineering, vol. 13, no. 2, pp. 180-187, 2017, doi:10.33762/eeej.2017.135282.

[11] Ammour B., Bouden T., and Amira-Biad S., "Multimodal biometric identification system based on the face and iris," In 2017 5th International Conference on Electrical Engineering-Boumerdes (ICEE-B), 2017, pp. 1-6, doi: 10.1109/ICEE-B.2017.8191981.

[12] Ammour B., Bouden T., and Boubchir L., "Face-iris multi-modal biometric system using multi-resolution LogGabor filter with spectral regression kernel discriminant analysis," IET Biometrics, vol. 7, no. 5, pp. 482-489, 2018, doi:10.1049/iet-bmt.2017.0251.

[13] Sujana S. and Reddy V. S. K., "Weighted Fusion Approach for Multi Modal Biometric Recognition System using Deep Networks," International Journal of Advanced Research in Engineering and Technology, vol. 11, no. 12, pp. 2282-2290, 2020, doi: 10.34218/ijaret.11.12.2020.216.

[14] Matin A., Mahmud F., Ahmed T., and Ejaz M. S., "Weighted score level fusion of iris and face to identify an individual," 2017 International Conference on Electrical, Computer and Communication Engineering (ECCE), 2017, pp. 1-4, doi: 10.1109/ECACE.2017.7912868.

[15] Azom V., Adewumi A., and Tapamo J. R., "Face and Iris biometrics person identification using hybrid fusion at feature and score-level," 2015 Pattern Recognition Association of South Africa and Robotics and Mechatronics International Conference (PRASA-RobMech), 2015, pp. 207-212, doi: 10.1109/RoboMech.2015.7359524.

[16] Huo G., Liu Y., Zhu X., Dong H., and He F., "Face-iris multimodal biometric scheme based on feature level fusion," Journal of Electronic Imaging, vol. 24, no. 6, 2015, doi: 10.1117/1.JEI.24.6.063020.

[17] Eskandari M. and Toygar Ö., "Selection of optimized features and weights on face-iris fusion using distance images," Computer Vision and Image Understanding, vol. 1, no. 137, pp. 63-75, 2015, doi: 10.1016/j.cviu.2015.02.011.

[18] Khiari-Hili N., Montagne C., Lelandais S., and Hamrouni K., "Quality dependent multimodal fusion of face and iris biometrics," In 2016 Sixth International Conference on Image Processing Theory, Tools and Applications (IPTA), 2016, pp. 1-6, doi: 10.1109/IPTA.2016.7820954.

[19] Minaee S., Abdolrashidi A., and Wang Y., "Face recognition using scattering convolutional network," 2017 IEEE signal processing in medicine and biology symposium (SPMB), 2017 Dec 2, pp. 1-6, doi: 10.1109/SPMB.2017.8257025.

[20] Sharifi O. and Eskandari M., "Optimal face-iris multimodal fusion scheme," Symmetry, vol. 8, no. 6, 2016, doi: $10.3390 /$ sym 8060048 .

[21] Ahmadi N., and Akbarizadeh G., "Hybrid robust iris recognition approach using iris image pre-processing, twodimensional gabor features and multi-layer perceptron neural network/PSO," Iet Biometrics, vol. 7, no. 2, pp. 153-162, 2017, doi: 10.1049/iet-bmt.2017.0041. 
[22] Ammour B., Bouden T., and Boubchir L., "Face-iris multimodal biometric system based on hybrid level fusion," 2018 41st International Conference on Telecommunications and Signal Processing (TSP), 2018, pp. 1-5, doi: 10.1109/TSP.2018.8441279.

[23] Dua M., Gupta R., Khari M., and Crespo R. G., "Biometric iris recognition using radial basis function neural network," Soft Computing, vol. 23, no. 22, pp. 11801-11815, 2019, doi: 10.1007/s00500-018-03731-4.

[24] Agarwal R., Singh Jalal A., and Arya K. V., "A multimodal liveness detection using statistical texture features and spatial analysis," Multimedia Tools and Applications, vol. 79, no. 11, pp. 1-25, Jan. 2020, doi: 10.1007/s11042-019-08313-6.

[25] Mustafa A. S., Abdulelah A. J., and Ahmed A. K.," Multimodal Biometric System Iris and Fingerprint Recognition Based on Fusion Technique," International Journal of Advanced Science and Technology, vol. 29, no. 3, pp. 7423-7432, 2020.

[26] Mansoura L., Noureddine A., Assas O., and Yassine A., "Biometric recognition by multimodal face and iris using FFT and SVD methods With Adaptive Score Normalization," 2019 4th World Conference on Complex Systems (WCCS), 2019, pp. 1-5, doi: 10.1109/ICoCS.2019.8930748.

[27] Raja J., Gunasekaran K., and Pitchai R., "Prognostic evaluation of multimodal biometric traits recognition based human face, finger print and iris images using ensembled SVM classifier," Cluster Computing, vol. 22, no. 1, pp. 215-28, 2019, doi: 10.1007/s10586-018-2649-2.

[28] Ammour B., Boubchir L., Bouden T., and Ramdani M., "Face-Iris Multimodal Biometric Identification System," Electronics, vol. 9, no. 1, p. 85, 2020 Jan, doi: 10.3390/electronics9010085.

[29] Sireesha V. and Reddy S. R., "Two Levels Fusion Based Multimodal Biometric Authentication Using Iris and Fingerprint Modalities," International Journal of Intelligent Engineering and Systems, vol. 9, no. 3, pp. 21-35, 2016, doi: 10.22266/IJIES2016.0930.03.

[30] Chaudhary S. and Nath R., "A robust multimodal biometric system integrating iris, face and fingerprint using multiple SVMs," International Journal of Advanced Research in Computer Science, vol. 7, no. 2, 2016, doi: 10.26483/ijarcs.v7i2.2647.

[31] Kabir W., Ahmad M. O., and Swamy M. N., "A multi-biometric system based on feature and score level fusions," IEEE Access, vol. 7, pp. 59437-59450, 2019, doi: 10.1109/ACCESS.2019.2914992.

[32] Patil A. P. and Bhalke D. G., "Fusion of fingerprint, palmprint and iris for person identification," 2016 International Conference on Automatic Control and Dynamic Optimization Techniques (ICACDOT), 2016, pp. 960-963, doi: 10.1109/ICACDOT.2016.7877730.

[33] Toygar Ö., Alqaralleh E., and Afaneh A.," Person Identification Using Multimodal Biometrics under Different Challenges," Human-Robot Interaction-Theory and Application, pp. 81-96, 2018, doi: 10.5772/intechopen.71667.

[34] Ali M. M., Mahale V. H., Yannawar P., and Gaikwad A. T., "Fingerprint recognition for person identification and verification based on minutiae matching," 2016 IEEE 6th International Conference on Advanced Computing (IACC), 2016, pp. 332-339, doi: 10.1109/IACC.2016.69.

[35] Sarhan S., Alhassan S., and Elmougy S., "Multimodal biometric systems: a comparative study," Arabian Journal for Science and Engineering, vol. 42, no. 2, pp. 443-457, 2017, doi: 10.1007/s13369-016-2241-0.

[36] Gad R., El-Fishawy N., El-Sayed A. Y, and Zorkany M., "Multi-biometric systems: a state of the art survey and research directions," International Journal of Advanced Computer Science and Applications (IJACSA), vol. 6, no. 6, 2015, doi: 10.14569/IJACSA.2015.060618.

[37] Sanjekar P. S. and Patil J. B., "An overview of multimodal biometrics," Signal \& Image Processing, vol. 4, no. 1, pp. 57-64, 2013 Feb 1, doi: 10.5121/sipij.2013.4105.

[38] Jamdar C. and Boke A., "Multimodal biometric identification system using fusion level of matching score level in single modal to multi-modal biometric system," 2017 International Conference on Energy, Communication, Data Analytics and Soft Computing (ICECDS), 2017, pp. 2277-2280, doi: 10.1109/ICECDS.2017.8389858.

[39] Oloyede M. O. and Hancke G. P., "Unimodal and multimodal biometric sensing systems: a review," IEEE Access, vol. 4, pp. 7532-55, 2016, doi: 10.1109/ACCESS.2016.2614720.

[40] Kaur G, Bhushan S., and Singh D., "Fusion in multimodal biometric system: A review," Indian Journal of Science and Technology, vol. 10, no. 28, pp. 1-6, 2017, doi: 10.17485/ijst/2017/v10i19/114382.

[41] Friedman L. and Komogortsev O. V., "Assessment of the effectiveness of seven biometric feature normalization techniques," IEEE Transactions on Information Forensics and Security, vol. 14, no. 10, pp. 2528-2536, 2019, doi: 10.1109/TIFS.2019.2904844.

[42] Sultana M., Paul P. P., and Gavrilova M. L., "Social behavioral information fusion in multimodal biometrics," IEEE Transactions on Systems, Man, and Cybernetics: Systems, vol. 48, no. 12, pp. 2176-2187, 2017, doi: 10.1109/TSMC.2017.2690321.

[43] Walia G. S., Singh T., Singh K., and Verma N., "Robust multimodal biometric system based on optimal score level fusion model, "Expert Systems with Applications, vol. 116, pp. 364-376, 2019, doi: 10.1016/j.eswa.2018.08.036.

[44] Kumar A. and Shekhar S., "Personal identification using multibiometrics rank-level fusion," IEEE Transactions on Systems, Man, and Cybernetics, Part C (Applications and Reviews), vol. 41, no. 5, pp. 743-752, 2010, doi: 10.1109/TSMCC.2010.2089516.

[45] Li J., Qiu T., Wen C., Xie K., and Wen F. Q., "Robust face recognition using the deep C2D-CNN model based on decision-level fusion," Sensors, vol. 18, no. 7, pp. 2080, 2018, doi: 10.3390/s18072080.

[46] Mehdi Cherrat E., Alaoui R., and Bouzahir H., "Convolutional neural networks approach for multimodal biometric identification system using the fusion of fingerprint, finger-vein and face images," PeerJ Computer Science, vol. 6 , p. e248, 2020, doi: 10.7717/peerj-cs.248. 
[47] Lahat D., Adali T., and Jutten C., "Multimodal Data Fusion: An Overview of Methods, Challenges, and Prospects," Proceedings of the IEEE, vol. 103, no. 9, Sept. 2015, pp. 1449-1477, doi: 10.1109/JPROC.2015.2460697.

[48] Garg S. N., Vig R., and Gupta S., "A survey on different levels of fusion in multimodal biometrics," Indian Journal of Science and Technology, vol. 10, no. 44, 2017, doi: 10.17485/ijst/2017/v10i44/120575.

[49] Walia G. S., Singh T., Singh K., and Verma N., "Robust multimodal biometric system based on optimal score level fusion model," Expert Systems with Applications, vol. 116, pp. 364-76, 2019, doi: 10.1016/j.eswa.2018.08.036.

[50] Anand A., Labati R. D., Genovese A., Munoz E., Piuri V., and Scotti F., "Age estimation based on face images and pre-trained convolutional neural networks," 2017 IEEE Symposium Series on Computational Intelligence (SSCI), 2017, pp. 1-7, doi: 10.1109/SSCI.2017.8285381.

\section{BIOGRAPHIES OF AUTHORS}

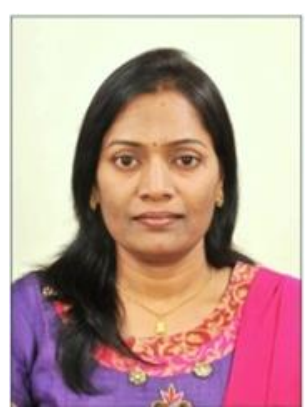

Sujana Surineni is pursuing a Ph.D. in the area of biometrics at Jawaharlal Nehru Technological University, Hyderabad under the supervision of Dr. VSK Reddy. She has received a B.Tech (ECE) and M.Tech (DSCE) degrees from Jawaharlal Nehru Technological University, Hyderabad, India. She is currently working as an Assistant Professor in the Department of ECE, Vardhaman College of Engineering, Hyderabad. Her research interests include Computer Vision, MachineLearning, and Image Processing with a focus on Multimodal Biometrics.

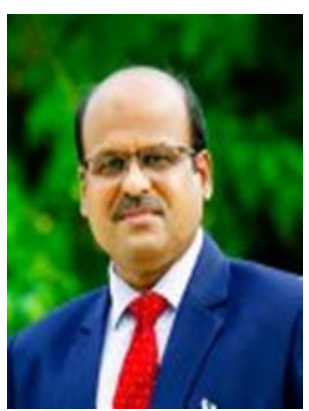

Dr. V. S. K. Reddy, Vice-Chancellor, Malla Reddy University has experience of more than 25 years in Teaching and Industry put together. He is an alumnus of the Indian Institute of Technology, (IIT) Kharagpur, obtained his Ph.D. and he is versatile in multidisciplinary specializations in Signal Processing \& Communications as well as Computer Science Engineering. His laurels include more than 150 Publications in reputed National and International Conferences and Journals. He is a fellow of IETE, a Life Member of ISTE, a Member of IEEE, and a member of CSI. He was a member of the Board of Studies for JNTU Hyderabad. He is the Education Fellowship Awardee from British Council, the UK to visit England, Scotland, Wales, and Ireland Countries for analyzing the education system in the UK. He is an accomplished academician, renowned speaker, and visiting professor to various Universities in UK and Malaysia. 